\title{
Facilitando la autorregulación del aprendizaje en el diseño de sistemas digitales
}

David de Andrés Martínez ${ }^{\mathrm{a}}$

aUniversitat Politècnica de València, ddandres@disca.upv.es.

\section{Abstract}

The software development paradigm students are used to is completely different to the one that must be followed when facing the challenge of specifying and implementing digital systems (hardware). The change in the perspective of how to tackle practical problems in this context could be quite troublesome for students, specially when they lack tools that ease the selfregulation of their cognitive processes. This paper adapts the self-regulated learning model by Winne and Hadwin to the context of designing digital systems. This model is based on continuous monitoring and controlling one's learning process. Scaffolding and prompting techniques are essential to facilitate the development of strategies for self-regulation. This paper presents the defined planning, its initial deployment, and the partial results obtained.

Keywords: metacognition, self-regulation, scaffolding, MSLQ, digital systems.

\section{Resumen}

El paradigma al que el alumnado está acostumbrado para el desarrollo de software cambia completamente cuando debe enfrentarse al reto de especificar e implementar sistemas digitales (hardware). Este cambio de concepción y forma de abordar problemas prácticos en este contexto puede representar un escollo importante para el alumnado, especialmente cuando no dispone de herramientas que faciliten la autorregulación de su proceso cognitivo. Esta comunicación presenta una adaptación al contexto del diseño de sistemas digitales del modelo de aprendizaje autorregulado de Winne y Hadwin, basado en la monitorización continua y el control del propio proceso de aprendizaje. El uso de técnicas de andamiaje y apunte son esenciales para facilitar el desarrollo de estrategias de autorregulación. Se presenta la planificación realizada, su despliegue inicial y los resultados parciales obtenidos hasta la fecha.

Palabras clave: metacognición, autorregulación, andamiaje, MSLQ, sistemas digitales. 


\section{Introducción}

La Recomendación del Parlamento Europeo y del Consejo de 18 de diciembre de 2006 sobre las competencias clave para el aprendizaje permanente (European Parliament, Council of the European Union, 2006) indica que, "dados los nuevos retos que la globalización sigue planteando a la Unión Europea, cada ciudadano requerirá una amplia gama de competencias para adaptarse de modo flexible a un mundo que está cambiando con rapidez y muestra múltiples interconexiones. En su doble función — social y económica -, la educación y la formación deben desempeñar un papel fundamental para garantizar que los ciudadanos europeos adquieran las competencias clave necesarias para poder adaptarse de manera flexible a dichos cambios". Entre las competencias clave que esta recomendación identifica se incluye aprender a aprender, que posibilita la adquisición, procesamiento y asimilación de nuevos conocimientos y capacidades, así como la búsqueda y uso de orientaciones. A partir de esta recomendación, los diferentes estados miembros deben desarrollar la oferta de competencias clave para todos en el contexto de sus estrategias de aprendizaje permanente.

En concreto, en España se extienden estas estrategias a las enseñanzas universitarias oficiales a través del Real Decreto 1393/2007 de 29 de octubre (Ministerio de Educación y Ciencia, 2007), donde se indica que se "impulsa un cambio en las metodologías docentes, que centra el objetivo en el proceso de aprendizaje del estudiante, en un contexto que se extiende ahora a lo largo de la vida" y, por tanto, "los planes de estudios conducentes a la obtención de un título deberán tener en el centro de sus objetivos la adquisición de competencias por parte de los estudiantes". La Universitat Politècnica de València (UPV), a través de su proyecto institucional de competencias transversales (Universitat Politècnica de València, 2012), sintetiza el perfil competencial que todo alumno egresado de la UPV debería adquirir. Este perfil incluye, como competencia número 11, el "aprendizaje permanente".

Así pues, queda patente la necesidad para la sociedad y el interés en todos los niveles educativos, de desarrollar en el aprendiente la capacidad de "aprender a aprender". Este concepto está íntimamente ligado al de metacognición (Schraw \& Mosham, Metacognitive Theories, 1995), entendido como la capacidad de las personas para reflexionar sobre sus procesos de pensamiento y la forma en que aprenden., aumentando así su eficacia, su rendimiento y el control sobre sí mismo (autorregulación) (Hacker, 1998).

Dentro de este contexto, se ubica la asignatura Diseño de Sistemas Digitales (Universitat Politècnica de València, 2018), optativa de la Rama de Ingeniería de Computadores, del cuatrimestre 3B del Grado de Ingeniería Informática de la UPV, con un número medio de 38 alumnos matriculados, durante los últimos 5 años. Los contenidos de la asignatura no son especialmente difíciles, pero el reto de diseñar e implementar circuitos utilizando herramientas de desarrollo profesionales, supone una rotura del paradigma tradicional de desarrollo que los alumnos han seguido en sus primeros años en la titulación. La especificación de circuitos requiere de su descripción por medio de lenguajes de descripción de hardware, cuya utilización es diametral opuesta a los lenguajes de programación para la especificación de software, a los que los alumnos están acostumbrados. Asimismo, los procesos involucrados en el flujo de diseño son diferentes y requieren de una mayor sintonización y precisión para alcanzar un producto final competitivo. Todo ello, unido a la 
falta de autorregulación que los alumnos manifiestan de formas generalizada durante los últimos años, hace que la dedicación de los alumnos a la realización de las prácticas sea muy alta, pero en momentos puntuales, y que los resultados obtenidos estén por debajo de lo esperado (Winne \& Nesbit, Supporting Self-Regulated Learning with Cognitive Tools, 2009):

- No pueden juzgar adecuadamente lo que saben: La dificultad de los conceptos teóricos y de las prácticas es incremental, por lo que es común el caso de alumnos que creen dominar la asignatura porque han conseguido superar las primeras prácticas, aunque no sobradamente, y que luego no saben cómo abordar prácticas sucesivas debido a que no han enfocado de forma adecuada su trabajo y estudio. Como no son capaces de reconocer su desconocimiento, no pueden desplegar estrategias adecuadas para su resolución o buscar ayuda efectiva.

- No conocen herramientas para estudiar de forma efectiva: Simples estrategias como la toma de notas en clase, la relectura del materal, la realización de resúmenes y/o diagramas, son grandes desconocidos para gran parte del alumnado, que confía en las transparencias disponibles y en las fotos tomadas de la pizarra. Esto dificulta que el alumnado pueda autorregular correctamente su aprendizaje, ya que no conoce estrategias básicas y/o alternativas de aprendizaje.

- Su sistema de creencias puede impedir una autorregulación efectiva: Aspectos clave como que el aprendizaje requiere esfuerzo, se aprende de los errores, y lo importante es el porqué y no el resultado, han sido relegados a un segundo plano en un mundo en el que la inmediatez es más importante que la cultura del esfuerzo.

- Deben enfrentarse a prejuicios humanos, heurísticos basados en probabilidades y razonamientos complejos: En general, el alumnado no dedica tiempo suficiente a las actividades desplegadas para mejorar su rendimiento y no toman nota de los pasos seguidos en el procedimiento, por lo que deben comenzar nuevamente un periodo de adaptación y descubrimiento al inicio de cada sesión práctica. Además, tienden a crear correlaciones falsas porque les dan más peso cuando ratifican sus preconcepciones que cuando las contradicen. En el caso de esta asignatura esto es crucial, ya que tienden a aplicar los procedimientos de desarrollo software ya aprendidos (e incorrecto en este contexto).

- Pueden no ser capaces de aprender de sus errores: A pesar de las actividades de la asignatura se planifican con dificultad y peso en la calificación creciente, y se facilita retroalimentación continua para facilitar el aprendizaje a partir de los errores, el alumnado experimenta una ansiedad y estrés creciente al enfrentarse a sus errores, lo que provoca que se fijen objetivos menos ambiciosos y abandono temprano

- No buscan ayuda de forma efectiva: El alumnado no suele buscar ayuda profesional, como podría ofrecer el Instituto de Ciencias de la Educación de la UPV, o con mayor conocimiento de estrategias apropiadas al contexto, como el profesorado de la asignatura. Sin embargo, suele acudir a sus iguales, especialmente alumnos que han cursado la asignatura anteriormente, que pueden no constituir una fuente fiable. 
Por ello, es necesario dotar a los aprendientes de la formación y las herramientas necesarias para superar estas barreras y alcanzar los niveles de metacognición y autorregulación requeridos de acuerdo con la etapa de su formación en curso.

\section{Modelos de autorregulación del aprendizaje}

La autorregulación del aprendizaje incluye todos aquellos aspectos cognitivos, metacognitivos, comportamentales, motivacionales y emotivos/afectivos del aprendizaje, por lo que constituye la mayor área de investigación de la psicología educacional (Torrano, Fuentes, \& Soria, 2017). Los seis modelos principales de autorregulación del aprendizaje analizados por Panadero (2017) incluyen: i) la perspectiva sociocognitiva de Zimmerman (2000), que explica la interrelación de los procesos metacognitivos y motivacionales a nivel individual; ii) el modelo de procesamiento dual de Boekaerts (Boekaerts \& Corno, 2005), donde las emociones del aprendiente dirigen las estrategias desplegadas para alcanzar los objetivos de las tareas (top-down) o evitar un daño personal (bottom-up); iii) el modelo de autorregulación del aprendizaje de Winne y Hadwin (1998), que posiciona a los aprendientes en una situación activa, en la que gestionan su propio aprendizaje por medio de su monitorización y el uso de estrategias metacognitivas; iv) el modelo de Pintrich (2000), que da respuesta a un gran número de procesos de autorregulación del aprendizaje a partir de la cognición, motivación/afecto, comportamiento y contexto; v) el Metacognitive and Affective Model of Self-Regulated Learning (MASRL) de Efkides (2011), que clarifica y detalla la relación entre metacognición, motivación y afecto a través de la interacción entre el nivel Persona (características del aprendiente) y el nivel Tarea $x$ Persona (interacción entre las características del aprendiente y el tipo de tarea); y vi) el modelo Socially Shared Regulation of Learning (SSRL) (Hadwin, Järvelä, \& Miller, 2017), que explica la regulación en las características interactivas y sociales del aprendizaje.

De todos ellos, el modelo de autorregulación del aprendizaje de Winne y Hadwin (1998) ha sido ampliamente utilizado, especialmente en investigaciones relacionados con entornos de aprendizaje basados en computador. Por ello, ha sido seleccionado como el modelo de referencia para esta innovación. La Fig. 1 muestra las cuatro fases recursivas definidas en el modelo. Primeramente, los aprendientes generan conocimiento acerca de la tarea a realizar a partir de su definición. A continuación, deben establecer los objetivos y establecer un plan para su consecución. Seguidamente, deben poner en marcha aquellas acciones (estrategias y técnicas de estudio) que les permitan alcanzar estos objetivos. Finalmente, el aprendiente decide realizar cambios duraderos (adaptación) en sus motivaciones, creencias y estrategias para el futuro. 


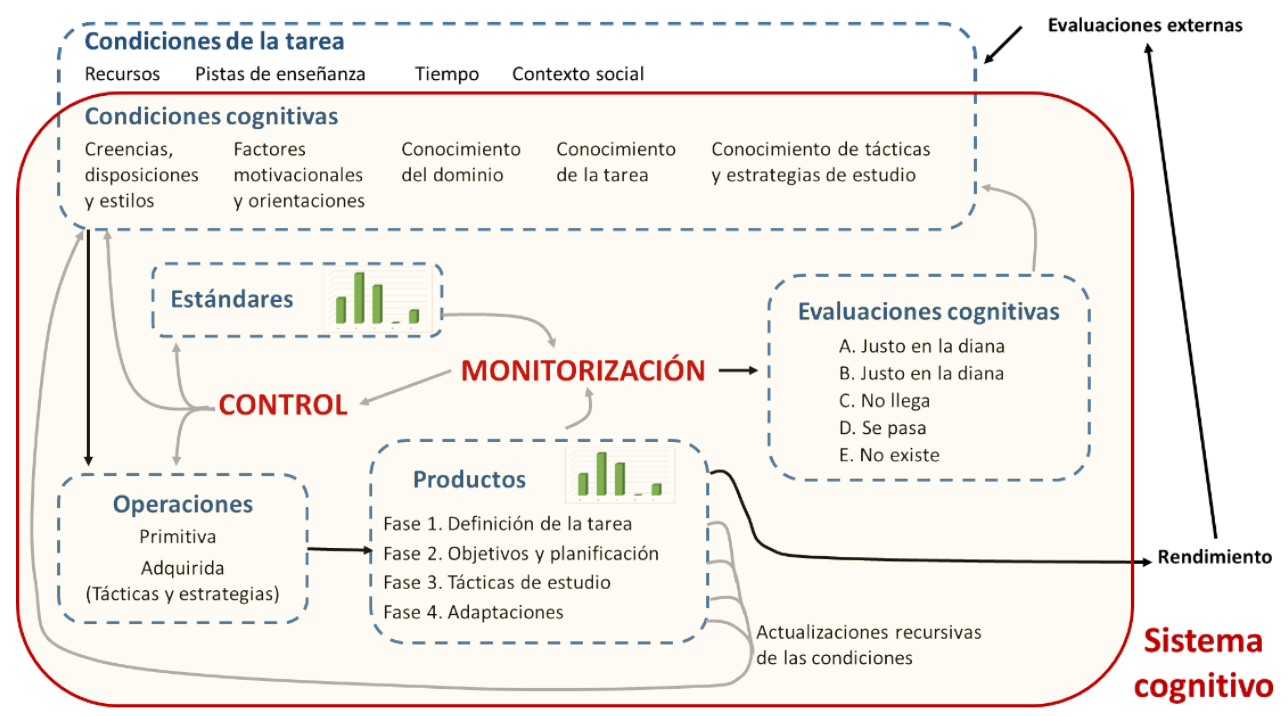

Fig. 1. Adaptado del modelo de aprendizaje autorregulado de Winne y Hadwind (1998)

Adicionalmente, este modelo identifica cincos aspectos a tener en consideración, identificados por el acrónimo COPES (del inglés, Conditions, Operations, Products, Evaluations, Standards). Las condiciones son aquellos recursos disponibles y las restricciones inherentes a la tarea (por ejemplo, tiempo limitado). Las operaciones incluyen aquellos procesos cognitivos, tácticas y estrategias utilizados por el aprendiente. Los productos son aquellas informaciones creadas por las operaciones. Las evaluaciones proporcionan retroalimentación acerca de la bondad de los productos con respecto a los estándares fijados. Los estándares son aquellos criterios utilizados para evaluar los productos.

En este modelo, la evaluación formativa y la autoevaluación desempeñan un rol fundamental para que los aprendientes puedan monitorizar los objetivos deseados y determinar qué estrategias favorecieron o frustraron su consecución (Fernández \& Jamet, 2017). Para que esta retroalimentación sea efectiva debe cumplir con los siguientes principios (Nicol \& Macfarlane-Dick, 2006): i) ayudar a clarificar qué se entiende por un buen rendimiento (objetivos, criterios y estándares esperados); ii) facilitar el desarrollo de la autoevaluación del aprendizaje; iii) proporcionar a los aprendientes información de alta calidad acerca de su aprendizaje; iv) incentivar el diálogo con el profesor y los compañeros acerca del aprendizaje; v) incentivar la autoestima y las creencias motivacionales positivas; vi) proporcionar oportunidades para reducir la distancia entre el rendimiento esperado y el alcanzado; y vii) proporcionar a los profesores información que puedan utilizar para mejorar la enseñanza.

La información necesaria para proporcionar esta retroalimentación se ha recogido tradicionalmente a través de autoinformes de los aprendientes, pero cada vez más se están utilizando protocolos de observación y trazas generadas por computador (Winne \& Perry, 2000). A pesar de su importancia, el confiar únicamente en estos autoinformes proporciona una visión sesgada de la autorregulación del aprendizaje, ya que los aprendientes basan sus respuestas en recuerdos imprecisos, experiencias sesgadas, contextos variados, y estrategias 
que conocen o creen efectivas en lugar de las que realmente han utilizado (McCardle \& Hadwin, 2015). Las tecnologías de la información y las comunicaciones (TIC) pueden resultar de gran ayuda, por un lado, para obtener trazas sensibles a los procesos cognitivos desplegados, a las tareas realizadas y los tiempos empleados, y por otro lado, para involucrar a los aprendientes en una autorregulación de su aprendizaje más efectiva, proporcionando herramientas de soporte a la identificación de qué debe aprenderse y cómo lograrlo (Winne \& Nesbit, Supporting Self-Regulated Learning with Cognitive Tools, 2009). Dos factores críticos en esta innovación son i) los datos que pueden obtenerse de forma automática, y ii) cómo analizar estos datos y mostrarlos a los aprendientes a modo de retroalimentación.

\section{Objetivos}

El objetivo que se persigue es la definición de una estrategia que facilite al alumnado la autorregulación de su aprendizaje, siguiendo el modelo de Winne y Hadwin, para mejorar la consecución de los objetivos de aprendizaje y desarrollar su capacidad de aprender a aprender, en el contexto del diseño de sistemas digitales. Para conseguirlo, se plantean las siguientes vías:

- En el modelo de Winne y Hadwin las condiciones cognitivas del alumnado no pueden ser controladas, obviamente, pero las condiciones de la tarea sí que dependen del profesor. Por ello, se modificarán los boletines de prácticas de la asignatura para identificar claramente los resultados de aprendizaje que se espera alcanzar con cada una de ellas. Esto permitirá a los alumnos definir las tareas, plantear sus objetivos y seleccionar las estrategias necesarias para su consecución.

- Se desplegará un sistema de monitorización automático que permitirá obtener trazas de todas las acciones realizadas por los alumnos en el sistema, así como de todos los errores que vayan apareciendo durante el desarrollo de las prácticas. El análisis automático de esta información permitirá el envío de nuevos prompts para guiar al alumnado y facilitar que alcance los objetivos de aprendizaje esperados en cada práctica. Asimismo, se proporcionarán información acerca de aquellos errores comunes y recurrentes.

- A fin de que el alumnado pueda monitorizar y evaluar su progreso para realizar las adaptaciones necesarias en vistas a mejorar su aprendizaje, es necesario que disponga de estándares que definan como evaluar el nivel de consecución de los objetivos de las tareas. Por ello, se definirán claramente las referencias que se utilizarán como guía de evaluación (posiblemente a través de rúbricas) para cada una de las prácticas, lo que facilitará la autoevaluación por parte del alumnado. Asimismo, se proporcionará retroalimentación detallada por parte del profesor acerca de la evaluación realizada de cada una de las prácticas realizadas.

- Se incorporarán prompts (apuntes - guías en la dirección correcta) en aquellos boletines en los que se estime conveniente para guiar a los alumnos en la autorregulación de su aprendizaje, especialmente los correspondientes a las primeras prácticas. Estos prompts se proporcionarán al finalizar cada una de las prácticas, junto con la retroalimentación, para facilitar la reflexión del alumnado y la adaptación de sus procesos cognitivos al contexto del diseño de sistemas digitales. 


\section{Desarrollo de la innovación}

Los participantes en esta innovación son los 42 alumnos matriculados en la asignatura Diseño de Sistemas Digitales del Grado de Ingeniería Informática de la Universitat Politècnica de València en el curso académico 2018-2019. Su despliegue, que se encuentra actualmente en desarrollo al tratarse de una asignatura de segundo cuatrimestre, consta de diversos elementos que se describen a continuación.

\subsection{Motivated Strategies for Learning Questionnaire}

Entre todos los instrumentos validados que permiten evaluar la autorregulación del aprendizaje, podemos destacar el cuestionario Motivated Strategies for Learning Questionnaire (MSLQ) (Pintrich, Smith, García, \& McKeachie, 1991), que ha sido ampliamente utilizado en todo el mundo. Este cuestionario consta de 81 ítems, con respuesta en escala Likert de 1 a 7 (not at all true for me - very true for me) que pretende medir la motivación y estrategias de aprendizaje del aprendiente. La motivación (31 ítems) se divide en componentes de valor (orientación a objetivos intrínsecos, orientación a objetivos extrínsecos, valor de la tarea), de expectativa (control de creencias de aprendizaje, autoeficacia para el aprendizaje y el rendimiento) y afectivos (ansiedad ante un examen). Las estrategias de aprendizaje (50 ítems) se dividen en cognitivas y metacognitivas (ensayo, elaboración, organización, pensamiento crítico, autorregulación metacognitiva), gestión de recursos (tiempo y entorno de estudio, regulación del esfuerzo, aprendizaje entre pares, búsqueda de ayuda). Este cuestionario se ha implementado por medio de Google Forms (ver Fig. 2) para facilitar su distribución, recogida de la información y análisis de los datos.

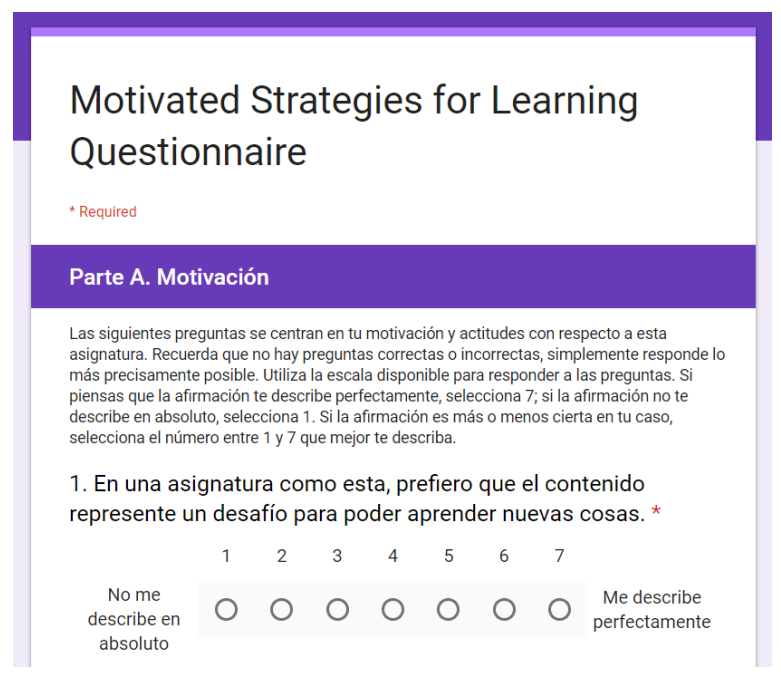

Fig. 2. Traducción y adaptación del cuestionario MSLQ (Pintrich, Smith, García, \& McKeachie, 1991)

Se ha pasado el cuestionario, a modo de pre-test, a los participantes antes del inicio de las sesiones prácticas. El cuestionario se volverá a completar, a modo de post-test, una vez 
finalizadas las sesiones prácticas. Entre ambas medidas tendrán lugar las sesiones prácticas de la asignatura en las que se desplegarán las acciones destinadas a favorecer el desarrollo de la autorregulación del aprendizaje del alumnado. Los participantes se han identificado por medio de documento nacional de identida o pasaporte, con lo que es posible identificarlos y realizar una análisis pareado para comprobar la efectividad de las acciones desplegadas sobre las estrategias medidas en el cuestionario. Asimismo, se ha solicitado una serie de datos como sexo, año en el que se terminó bachillerato, si trabaja al mismo tiempo que estudia, cuántas asignaturas estudia al mismo tiempo y si es la primera vez que cursa la asignatura, para poder comprobar si tienen algún efecto significativo en las estrategias analizadas. Adicionalmente, han proporcionado una dirección de correo electrónico a la que se les enviarán los resultados obtenidos en estos cuestionarios.

\subsection{Andamiaje y apunte}

Todos los sistemas de aprendizaje proporcionan algún tipo de soporte (andamiaje, del inglés scaffolding), en el que los aprendientes interaccionan uno-a-uno con una persona con más experiencia (el profesor) que los guía para que desarrollen adecuadamente las funciones de autorregulación de su aprendizaje (Wood, Bruner, \& Ross, 1976). En las aulas modernas es prácticamente imposible que el profesor pueda interactuar de forma individual con todos los aprendientes y, es por ello, que las TIC pueden resultar de gran utilidad para soportar este andamiaje (Yen, y otros, 2018). En concreto, prompting (apuntar) es un método de instrucción para guiar y dar soporte a la autorregulación de los aprendientes en los procesos de resolución de problemas (Ifenthaler, 2012). Pueden presentarse como preguntas simples, sentencias incompletas, instrucciones a ejecutar o imágenes y gráficos. Estas guías pueden orientarse tanto para estimular las actividades cognitivas, metacognitivas, motivacionales o cooperativas de los aprendientes, como para soportar la monitorización y control del proceso de aprendizaje (Bannert, Sonnenberg, Mengelkamp, \& Pieger, 2015).

Así pues, los boletines de prácticas se han modificado para clarificar desde el principio los objetivos de aprendizaje a alcanzar con la actividad a desarrollar, incluir diferentes elementos que guían al alumnado a lo largo del proceso de especificación e implementación de los circuitos requeridos, e identificar los estándares con los que se evaluará la consecución de los objetivos planteados.

Por ejemplo, la actividad a desarrollar en la primera práctica consiste en especificar, en el lenguaje de descripción de hardware VHDL, e implementar en la placa de prototipado disponible en el laboratorio, un sistema que permita realizar la suma y/o resta de dos números de 4 bits y visualizar los datos de entrada y el resultado utilizando los displays de 7 segmentos disponibles en la placa. Los alumnos suelen centrar su atención en resolver el problema (el resultado final que deben entregar), no en cómo lo han resuelto (el proceso seguido para ello) o qué deberían haber aprendido en el proceso. Por ello, muchas veces no terminan de entender porqué unas aproximaciones son mejores que otras, cómo podrían mejorar su propuesta a partir del trabajo realizado, ni cómo reutilizar lo aprendido en posteriores prácticas. Así, el 
boletín de la práctica incluye ahora la descripción detallada de los objetivos de aprendizaje a alcanzar, lo que permite que el alumnado pueda juzgar si los ha alcanzado al finalizarla:

- Especificar componentes combinacionales por medio de sentencias de asignación concurrente (simple, condicional o con selección) en VHDL.

- Especificar componentes secuenciales mediante una descripción comportamental por medio de procesos en VHDL.

- Integrar componentes previamente diseñados por medio de una descripción estructural en VHDL.

- Definir componentes genéricos en VHDL que puedan reutilizarse en diseños posteriores.

- Verificar el correcto funcionamiento de los componentes especificados en VHDL mediante simulación.

- Especificar restricciones de localización de pines para permitir la implementación del diseño en una placa de prototipado determinada.

- Implementar el diseño especificado para una placa de prototipado determinada.

- Verificar el correcto funcionamiento del diseño sobre la placa de prototipado.

Adicionalmente, se han incluido anotaciones en el boletín de prácticas que pretenden guiar al alumno en el proceso, de tal forma que puedan familizarse con las aproximaciones más comunes para abordar el tipo de problemas que se plantean en este contexto. Por ejemplo, el reloj de la placa de prototipado funciona a una frecuencia demasiado elevada (100 MHz) para controlar la visaualización en los displays de 7 segmentos (deberían activar entre una vez cada milisegundo y una vez cada 16 milisegundos). Después de entender que deben generar un componente para dividir la frecuencia de funcionamiento, la mayor parte de los alumnos optan por utilizar la salida generada por este componente como señal de reloj de los componentes síncronos que deban funcionar con una frecuencia reducida. Esta es una opción viable, pero es una mejor opción de diseño el utilizar esta salida como señal de habilitación de los componentes síncronos. Otro aspecto crucial en el desarrollo de sistemas digitales es la especificación del sistema por medio de dibujos esquemáticos que muestren los componentes, sus entradas y salidas, y las conexiones establecidas entre ellos. El diagrama del sistema final a implementar en esta práctica puede verse en la Fig. 3.

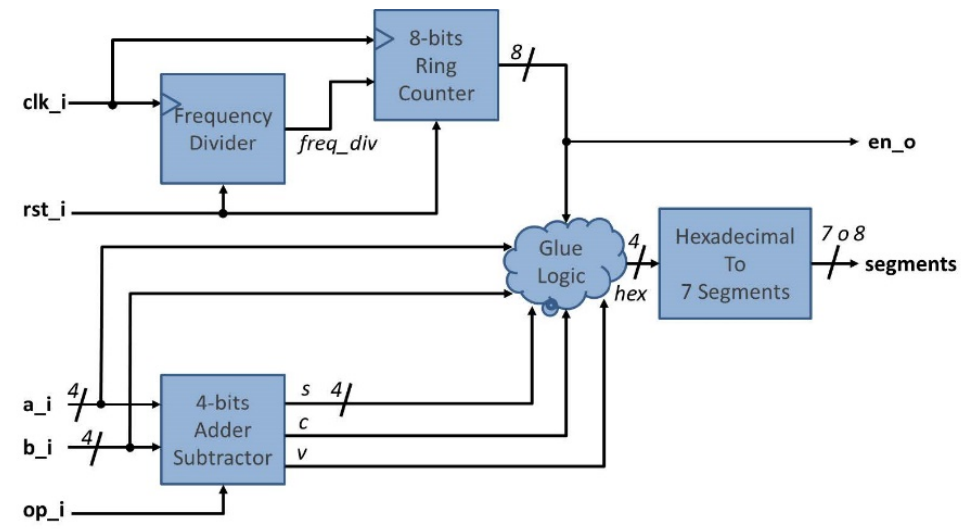

Fig. 3. Descripción esquemática que especifica los componentes a utilizar, su entradas, salidas e interconexión. 


\subsection{Monitorización automática}

El entorno de desarrollo utilizado en las sesiones prácticas es Vivado Design Suite - HLx Editions (Xilinx Inc., 2019), versión 2018.2, de Xilinx, dado que los dispositivos lógicos programables incluidos en las placas de prototipado disponibles en el laboratorio son de este fabricante.

Este entorno actúa como interfaz que aglutina, configura y lanza de forma sincronizada, multitud de herramientas diferentes para diseñar, implementar y verificar el circuito deseado. Todos los procesos pueden configurarse y lanzarse desde la consola integrada de comandos Tcl (Tool Command Language), pero la interfaz gráfica facilita estas operaciones y lanza los comandos necesarios en segundo plano. Los resultados e información proporcionada por los procesos ejecutados se muestran el ventana de Log. Adicionalmente, el entorno de desarrollo genera un fichero jou (journal) que almacena todos los comandos Tcl que se han lanzado durante la sesión, con su fecha y hora (timestamp), y otro fichero .log que almacena los resultados proporcionados por los procesos ejecutados, incluyendo los diversos problemas encontrados (información, advertencias, advertencias críticas y errores). La Tabla 1 muestra un extracto de un fichero. $\log$.

Tabla 1. Extracto de un fichero .log que recoge los problemas encontrados (en rojo) durante la síntesis del sistema

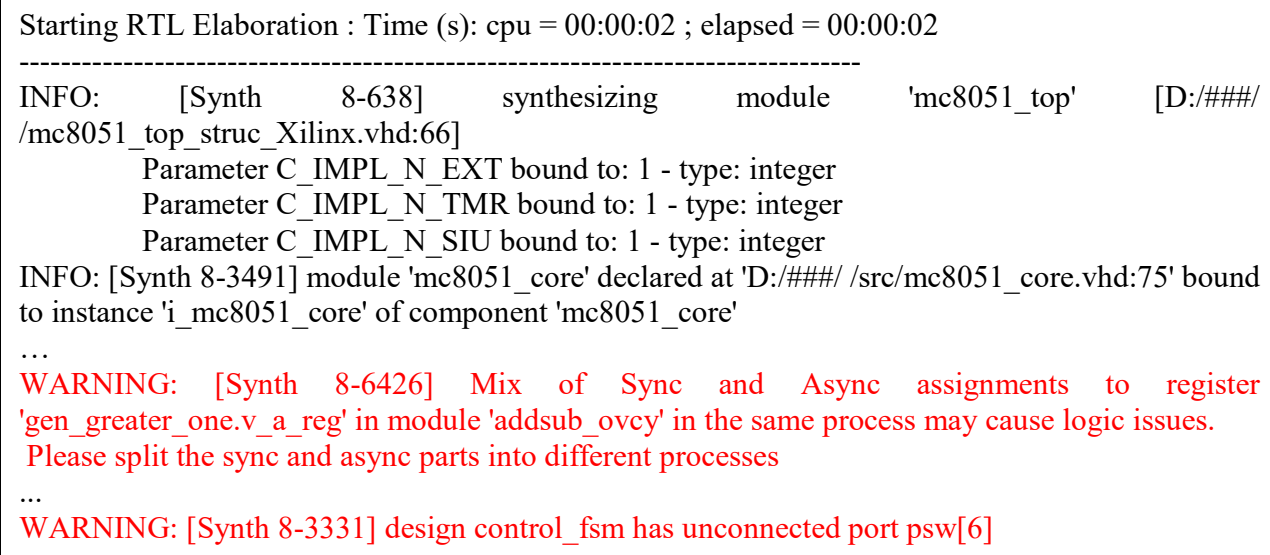

Se ha configurado la instalación realizada en las máquinas disponibles en el laboratorio de la asignatura, para guardar en un directorio una copia de los ficheros .jou y . $\log$ que se vayan generando. Estos ficheros, que pueden recuperse posteriormente para su análisis, identificarán al alumno que estaba trabajando en la máquina y permitirán determinar qué flujo de trabajo ha seguido, el instante de tiempo en el que lanzó cada proceso, y los problemas con los que se ha ido encontrando. Este análisis permitirá determinar aquellos problemas comunes que el alumnado se encuentra, en general, de tal forma que puedan prevenirse inicialmente por medio de anotaciones en el boletín de prácticas. Adicionalmente, dado que los informes que proporciona el entorno de desarrollo suelen ser ininteligibles por 
el alumnado, se desarrollará una página de preguntas más frecuentes, con guías que permitan seguir avanzando en el desarrollo de las prácticas sin necesidad de esperar a que el profesor acuda para la resolución del problema.

Actualmente se dispone de la instrumentación necesaria para la recogida de los ficheros, pero todavía se está trabajando en su análisis.

\section{$5 \quad$ Resultados}

Tal y como se ha indicado anteriormente, la innovación se encuentra en pleno desarrollo y aplicación, por lo que no se dispondrán de los resultados finales hasta que termine el cuatrimestre en curso. Sin embargo, sí que se dispone de los resultados del cuestionario MSLQ utilizado a modo de pre-test, y que se repetirá al finalizar la asignatura.

Del total de 42 alumnos que están cursando la asignatura, únicamente 23 accedieron a responder el cuestionario, lo que supone un margen de error del 13.9\%. Este efecto no puede mitigarse, dado las restricciones con las trabajamos en el aula y, a pesar de que se insistió a los alumnos en la utilidad del cuestionario y los beneficios que podrían derivarse de su aplicación, no se consiguió una mayor participación.

Como datos que posteriormente podrán utilizarse para comprobar su influencia en la autorregulación de los alumnos, podemos destacar que constestaron un $83 \%$ de hombres y un $17 \%$ de mujeres, que el $43.5 \%$ de los particpantes trabaja además de cursar sus estudios, y que el $34.8 \%$ ya ha cursado con anterioridad la asignatura.

Los valores medios y desviación típica de todos los participantes, para cada una de las dimensiones analizadas, se muestra en la Tabla 2.

Tabla 2. Valores medios y desviación típica globales de las dimensiones analizadas en el pre-test

\begin{tabular}{|c|c|c|c|}
\hline Escala & Componente & Ítem & $\begin{array}{l}\text { Media } \pm \\
\text { Desviación típica }\end{array}$ \\
\hline \multirow{6}{*}{ Motivación } & \multirow{3}{*}{ Valor } & Orientación a objetivo intrínseco & $5.53 \pm 1.42$ \\
\hline & & Orientación a objetivo extrínseco & $4.30 \pm 2.06$ \\
\hline & & Valor de la tarea & $5.72 \pm 1.32$ \\
\hline & \multirow[b]{2}{*}{ Expectativa } & Control de creencias de aprendizaje & $5.67 \pm 1.42$ \\
\hline & & $\begin{array}{l}\text { Autoeficacia para el aprendizaje y } \\
\text { rendimiento }\end{array}$ & $5.28 \pm 1.39$ \\
\hline & Afectivo & Ansiedad ante las pruebas & $3.76 \pm 2.11$ \\
\hline \multirow{9}{*}{$\begin{array}{l}\text { Estrategias } \\
\text { de } \\
\text { aprendizaje }\end{array}$} & \multirow{5}{*}{$\begin{array}{l}\text { Estrategias } \\
\text { cognitivas y } \\
\text { metacognitivas }\end{array}$} & Ensayo & $3.22 \pm 1.85$ \\
\hline & & Elaboración & $4.89 \pm 1.68$ \\
\hline & & Organización & $3.47 \pm 1.81$ \\
\hline & & Pensamiento crítico & $4.35 \pm 1.64$ \\
\hline & & Autorregulación metacognitiva & $4.56 \pm 1.76$ \\
\hline & \multirow{4}{*}{$\begin{array}{l}\text { Estrategias de } \\
\text { gestión de } \\
\text { recursos }\end{array}$} & Tiempo y entorno de estudio & $4.45 \pm 1.88$ \\
\hline & & Regulación del esfuerzo & $4.76 \pm 1.68$ \\
\hline & & Aprendizaje entre iguales & $3.52 \pm 1.86$ \\
\hline & & Búsqueda de ayuda & $3.90 \pm 1.99$ \\
\hline
\end{tabular}


En general, los valores medios obtenidos en el apartado de estrategias de aprendizaje son buenos, aunque hay que destacar valores menores de 4 en estrategías tales como el ensayo, la organización, el aprendizaje entre iguales y la búsqueda de ayuda. Adicionalmente, el resto de valores medio se encuentran no llegan al 5 en ningún caso, lo que unido a las desviaciones típicias alrededore de 1.8 parecen indicar que parte de los participantes no dispone de estrategias de aprendizaje adecuadas y deberían reforzarse. La efectividad de las acciones desplegadas en esta innovación no podrá comprobarse hasta la realización del post-test.

A cada uno de los participantes se les ha enviado por correo electrónico un informe con sus resultados del test, la media de la clase y los valores correspondientes a los 4 cuartiles. De esa forma, puede compararse con el resto de sus compañeros y decidir si precisa mejorar en algunas de las dimensiones estudiadas o incluso buscar ayuda en algún apartado específico. Además, el informe indica cómo intepretar los valores obtenidos y proporciona algunas sugerencias para mejorar las diferentes dimensiones. Un ejemplo del informe generado para un participante, en relación a la dimensión "Ensayo" se muestra en la Fig. 4.

\section{Estrategia cognitiva: Ensayo}

Esto es una medida de cuán a menudo utilizas estrategias de estudio como releer las notas de clase y las lecturas de la asignatura, y memorizar listas de palabras clave y conceptos. Una puntuación alta indica que utilizas estas estrategias bastante a menudo..

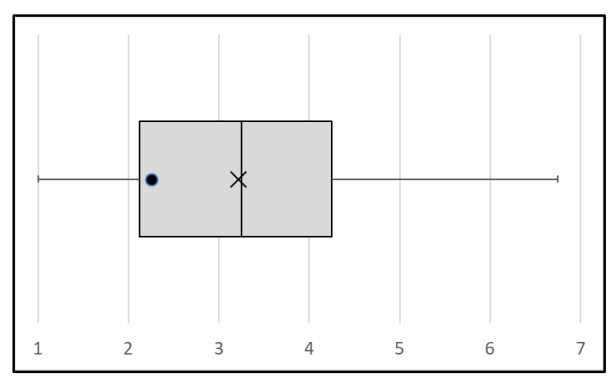

Recomendaciones: Lista los términos y temas importantes de la asignatura. Defínelos y repítelos en voz alta. Divide la lista en listas más pequeñas que se compongan de términos relacionados. Inventa imágenes o rimas que te ayuden a recordar estas listas. Genera baterías de preguntas para ayudarte a medir tu capacidad para recordarlas.

Fig. 4. Ejemplo de informe personalizado para un alumno en la dimensión "Ensayo". La cruz indica el valor medio de la clase y el punto el valor obtenido por el alumno

\section{Conclusiones}

La autorregulación del aprendizaje es esencial para poder desarrollar la competencia de aprender permanente, tan necesaria en la actualidad. El poder seleccionar y adaptar las estrategias de aprendizaje disponibles, resulta primordial para poder alcanzar los objetivos de aprendizaje de determinadas asignaturas, como el Diseño de Sistemas Digitales, en las 
que las concepciones y procedimientos ya aprendidos no facilitan, sino que dificultan, el progreso del alumnado.

Con el fin de mejorar las estrategias de aprendizaje y la autorregulación del alumnado, se ha puesto en marcha una innovación basada en el modelo de autorregulación del aprendizaje de Winne y Hadwin, basados en la monitorización continua y el control sobre los propios recursos cognitivos. Esta innovación incorpora los conceptos de andamiaje y apunte para guiar al alumnado en su progreso en las prácticas de la asignatura, así como una herramienta para la monitorización de las acciones realizadas y los problemas encontrados. Asimismo, el cuestionario MSLQ de Pintrich se ha utilizado como herramienta para evaluar las dimensiones relacionadas con la autorregulación del aprendizaje del alumnado al inicio de la asignatura y en su fase final, con lo que se podrá realizar una análisis de los efectos de las acciones llevadas a cabo.

Dado que esta innovación se encuentra actualmente en fase de desarrollo, se presenta su concepción, planificación y resultados iniciales. Se espera poder obtener resultados concluyentes a final del cuatrimestre y poder extrapolar la metodología y herramientas utilizadas a otras asignaturas de la misma titulación.

\section{Agradecimientos}

Los autores agradecen la financiación recibida por el Vicerrectorado de Estudios, Calidad y Acreditación de la Universitat Politècnica de València para desarrollar el Proyecto de Innovación y Mejora Educativa "Comunidades de Aprendizaje como servicios en la nube para el desarrollo y evaluación automática de Competencias Transversales y Objetivos Formativos específicos", con referencia B29.

\section{Referencias}

Bannert, M., Sonnenberg, C., Mengelkamp, C., \& Pieger, E. (2015). Short- and long-term effects of students' self-directed metacognitive prompts on navigation behavior and learning performance. Computers in Human Behavior, 52, 293-306. doi:http://dx.doi.org/10.1016/j.chb.2015.05.038

Boekaerts, M., \& Corno, L. (Abril de 2005). Self-Regulation in the Classroom: A Perspective on Assessment and Intervention. Applied Psychology, 199-231. doi:https://doi.org/10.1111/j.14640597.2005.00205.x

Efklides, A. (2011). Interactions of Metacognition With Motivation and Affect in Self-Regulated Learning: The MASRL Model. Educational Psychologist, 46(1), 6-25. doi:https://doi.org/10.1080/00461520.2011.538645

European Parliament, Council of the European Union. (2006). Recommendation of the European Parliament and of the Council of 18 December 2006 on key competences for lifelong learning. Recommendation. Recuperado el 22 de Marzo de 2019, de http://data.europa.eu/eli/reco/2006/962/oj

Fernández, J., \& Jamet, E. (2017). Extending the testing effect to self-regulated learning. Metacognition Learning, 12, 131-156. doi:10.1007/s11409-016-9163-9 
Hacker, D. J. (1998). Definitions and empirical foundations. En J. Dunlosky, A. C. Graesser, \& D. J. Hacker (Edits.), Metacognition in educational in a theory and practice (págs. 1-25). Mahwah, NJ: Lawrence Erlbaum Associates.

Hadwin, A. F., Järvelä, S., \& Miller, M. (2017). Self-regulation, co-regulation and shared regulation in collaborative learning environments. En D. H. Schunk, \& J. A. Greene (Edits.), Handbook of SelfRegulation of Learning and Performance. Routledge.

Ifenthaler, D. (2012). Determining the effectiveness of prompts for self-regulated learning in problemsolving scenarios. Educational Technology \& Society, 15(1), 38-52.

McCardle, L., \& Hadwin, A. F. (2015). Using multiple, contextualized data sources to measure learners' perceptions of their self-regulated learning. Metacognition and Learning, 10(1), 43-75. doi:https://doi.org/10.1007/s11409-014-9132-0

Ministerio de Educación y Ciencia. (2007). Real Decreto 1393/2007, de 29 de octubre, por el que se establece la ordenación de las enseñanzas universitarias oficiales. Boletín Oficial del Estado. Recuperado el 22 de Marzo de 2019, de https://www.boe.es/buscar/act.php?id=BOE-A-2007$18770 \& \mathrm{p}=20160603 \& \mathrm{tn}=1 \mathrm{\# a}$

Nicol, D. J., \& Macfarlane-Dick, D. (Abril de 2006). Formative assessment and self-regulated learning: A model and seven principles of good feedback practice. Studies in Higher Education, 31(2), 199-218.

Panadero, E. (28 de Abril de 2017). A Review of Self-regulated Learning: Six Models and Four Directions for Research. Frontiers in Psycology, 28. doi:https://doi.org/10.3389/fpsyg.2017.00422

Pintrich, P. R. (2000). The role of goal orientation in self-regulated learning. En M. Boekaerts, P. R. Pintrich, \& M. Zeidner (Edits.), Handbook of Self-Regulation (págs. 451-502). San Diego: Academic Press. doi:https://doi.org/10.1016/B978-012109890-2/50043-3

Pintrich, P. R., Smith, D. A., García, T., \& McKeachie, W. J. (1991). A manual for the use of the Motivated Strategies for Learning Questionnaire (MSLQ). University of Michigan, School of Education.

Schraw, G., \& Mosham, D. (12 de Marzo de 1995). Metacognitive Theories. Educational Psycology Review, 351-371. doi:https://doi.org/10.1007/BF02212307

Torrano, F., Fuentes, J., \& Soria, M. (2017). Aprendizaje autorregulado: estado de la cuestión y retos psicopedagógicos. Pefiles Educativos, XXXIX(156), 160-173.

Universitat Politècnica de València. (2012). Competencias Transversales. Recuperado el 22 de Marzo de 2019, de http://www.upv.es/contenidos/COMPTRAN/

Universitat Politècnica de València. (12 de Julio de 2018). Guía Docent 2018-2019. Recuperado el 22 de Marzo de 2019, de Diseño de Sistemas Digitales: https://gdocu.upv.es/alfresco/service/api/internal/shared/node/content/EzVmRDOWQ46sr_OoftKYk $\mathrm{w} ? \mathrm{a}=$ true.pdf

Winne, P. H., \& Hadwin, A. F. (1998). Studying as self-regulated learning. En D. J. Hacker, J. Dunlosky, \& A. C. Graesser (Edits.), Metacognition in educational theory (págs. 277-304). Nahwah, NJ: Lawrence Erlbaum Associates.

Winne, P. H., \& Nesbit, J. C. (2009). Supporting Self-Regulated Learning with Cognitive Tools. En D. J. Hacker, J. Dunlosky, \& A. C. Graesser (Edits.), Handbook of Metacognition in Education (págs. 259277). New York, USA: Taylor \& Francis.

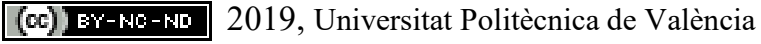


Winne, P. H., \& Perry, N. E. (2000). Measuring Self-Regulated Learning. En B. Monique, P. R. Paul, \& M. Zeidner (Edits.), Handbook of Self-Regulation (págs. 531-566). Academic Press. doi:https://doi.org/10.1016/B978-012109890-2/50045-7

Wood, D., Bruner, J. S., \& Ross, G. (1976). The role of tutoring in problem solving. Journal of CHild Psychology and Psychiatry, 89-100. doi:https://doi.org/10.1111/j.1469-7610.1976.tb00381.x

Xilinx Inc. (2019). Vivado Design Suite - HLx Editions. Recuperado el 23 de Marzo de 2019, de https://www.xilinx.com/products/design-tools/vivado.html

Yen, M.-H., Chen, S., Wang, C.-Y., Chen, H.-L., Hsu, Y.-S., \& Liu, T.-C. (22 de Marzo de 2018). A framework for self-regulated digital learning (SRDL). Journal of Computer Assisted Learning, 10. doi:https://doi.org/10.1111/jcal.12264

Zimmerman, B. J. (2000). Attaining Self-Regulation: A Social Cognitive Perspective. En M. Boekaerts, P. R. Pintrich, \& M. Zeidner (Edits.), Handbook of Self-Regulation (págs. 13-39). San Diego: Academic Press. doi:https://doi.org/10.1016/B978-012109890-2/50031-7 